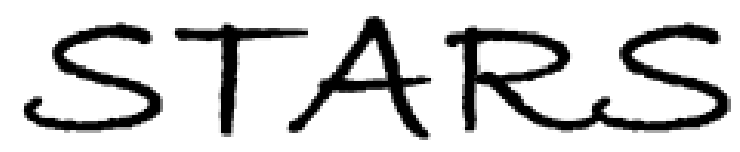

University of Central Florida

STARS

$1-1-1993$

\title{
Demonstration Of Demultiplexing With A Rocking Filter Fiber
}

\author{
C. G. Krautschik \\ University of Central Florida \\ P/ Wigley \\ University of Central Florida \\ G. I. Stegeman \\ University of Central Florida \\ R. H. Stolen
}

Find similar works at: https://stars.library.ucf.edu/facultybib1990

University of Central Florida Libraries http://library.ucf.edu

This Article is brought to you for free and open access by the Faculty Bibliography at STARS. It has been accepted for inclusion in Faculty Bibliography 1990s by an authorized administrator of STARS. For more information, please contact STARS@ucf.edu.

\section{Recommended Citation}

Krautschik, C. G.; Wigley, P/; Stegeman, G. I.; and Stolen, R. H., "Demonstration Of Demultiplexing With A Rocking Filter Fiber" (1993). Faculty Bibliography 1990s. 817.

https://stars.library.ucf.edu/facultybib1990/817

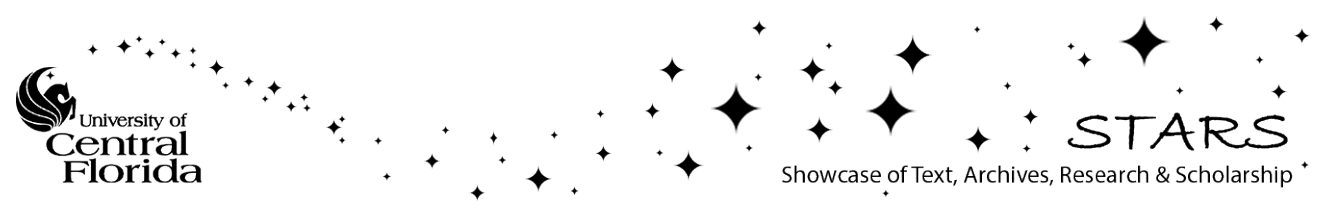




\section{Demonstration of demultiplexing with a rocking filter fiber}

Cite as: Appl. Phys. Lett. 63, 860 (1993); https://doi.org/10.1063/1.109881

Submitted: 22 February 1993 . Accepted: 28 May 1993. Published Online: 04 June 1998

C. G. Krautschik, P. Wigley, G. I. Stegeman, and R. H. Stolen

\section{ARTICLES YOU MAY BE INTERESTED IN}

Phase controlled all-optical switching in rocking filter fibers

Applied Physics Letters 61, 1751 (1992); https://doi.org/10.1063/1.108416

Characterization of photoinduced birefringence change in optical fiber rocking filters

Applied Physics Letters 68, 900 (1996); https://doi.org/10.1063/1.116224
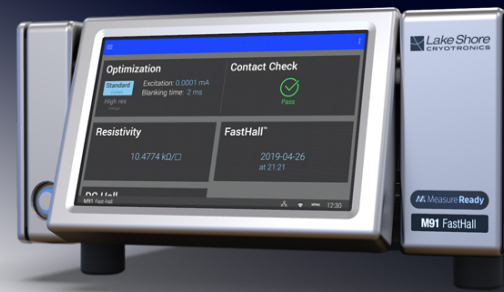

\section{Measure Ready M91 FastHall ${ }^{\text {Th }}$ Controller}

A revolutionary new instrument for complete Hall analysis

See the video 0 


\title{
Demonstration of demultiplexing with a rocking filter fiber
}

\author{
C. G. Krautschik, P. Wigley, and G. I. Stegeman \\ CREOL, University of Central Florida, Orlando, Florida 32826
}

\author{
R. H. Stolen \\ AT\&T Bell Laboratories, Holmdel, New Jersey 07733
}

(Received 22 February 1993; accepted for publication 28 May 1993)

\begin{abstract}
We show that the presence of a strong pump beam detuned from the center wavelength of a rocking filter fiber causes a signal beam at the filter resonance frequency to switch between polarization states. This process is used to switch out a pulse from a pulse train.
\end{abstract}

A very powerful application of all-optical phenomena in waveguides is to the demultiplexing of serial data. The goal, as indicated in Fig. 1, is to use an optical control pulse to switch specific pulses out of a serial data stream. When the control pulse overlaps a signal pulse in a waveguide, it modifies one of the signal beam's properties, typically its phase, polarization, or group velocity. This allows a particular signal to be isolated from the others either in space, polarization, or time. In fibers, this operation has previously been achieved by changing the signal plane of polarization by $90^{\circ}$ and discriminating with polarizers, by changing the signal phase and hence interference condition in a nonlinear loop mirror and by changing the group velocity and hence arrival time of a signal soliton pulse via an orthogonally polarized, copropagating soliton pulse. ${ }^{1-5} \mathrm{De}-$ multiplexing of $50 \mathrm{~Gb} / \mathrm{s}$ signals has already been reported. ${ }^{1}$ In this letter we demonstrate a novel demultiplexer that relies on a pump-pulse interaction in rocking rotator filter fibers.

A rocking filter fiber rotates over a limited range of wavelengths the plane of polarization of a beam incident along one of the fiber mode axes. ${ }^{6}$ Optimum rotation is obtained at the wavelength $\lambda_{c}$ when the fiber birefringence beat length $L_{b}\left(=2 \pi / \lambda_{c}\left|n_{x}-n_{y}\right|\right)$ equals the twist period $L_{t}$ induced in the fiber by periodically twisting the axes of the fiber during the drawing process. After each period the polarization rotates by a small angle, of the order of $1^{\circ}$, and after many periods the net angle can be many $\pi$. The length required for a $90^{\circ}$ rotation, i.e., between orthogonal polarizations, is called $L_{c}$. For wavelengths $\lambda$ different from $\lambda_{c}$, the process is detuned in wave vector by

$$
2 \delta=\frac{2 \pi\left|n_{x}-n_{y}\right|}{\lambda}-\frac{2 \pi}{L_{t}}=2 \pi\left(n_{x}-n_{y}\right) \frac{\lambda_{c}-\lambda}{\lambda^{2}} .
$$

The net rotation takes on a $\sin ^{2} \psi / \psi^{2}$ response, where $\psi=\left(\kappa^{2}+\delta^{2}\right)^{1 / 2} z$ after a distance $z$. Here, $\kappa=\pi / 2 L_{c}$.

If a second high intensity pump beam is present whose wavelength falls outside the bandwidth of the rocking rotator filter, the fiber birefringence at the signal wavelength changes via cross-phase modulation so that

$$
n_{x, y}\left(\lambda_{s}\right) \rightarrow n_{x, y}\left(\lambda_{s}\right)+\gamma\left[2 P_{x, y}\left(\lambda_{p}\right)+\frac{2}{3} P_{y, x}\left(\lambda_{p}\right)\right],
$$

where $\quad \gamma=n_{2} \omega / c A_{\text {eff }}{ }^{7} \quad$ Here, $A_{\text {eff }}=\left\langle\left|\psi_{p}\right|^{2}\right\rangle\left\langle\left|\psi_{s}\right|^{2}\right\rangle /$ $\left\langle\left|\psi_{p}\right|^{2}\left|\psi_{s}\right|^{2}\right\rangle$ with $\left\langle\left|\psi_{i}\right|^{2}\right\rangle=\iint\left|\psi_{i}(x, y)\right|^{2} d x d y$, where $\psi_{i}(x, y)$ is the transverse modal field profile at the wave- length $\lambda_{i}$. Assuming for simplicity that $n_{x}>n_{y}$ with $\gamma>0$, and substituting Eq. (2) into Eq. (1), the detuning now becomes

$$
\begin{aligned}
2 \delta= & 2 \pi\left[n_{x}\left(\lambda_{s}\right)-n_{y}\left(\lambda_{s}\right)\right] \frac{\left(\lambda_{c}-\lambda\right)}{\lambda_{c}^{2}} \\
& +\frac{4}{3} \gamma\left[P_{x}\left(\lambda_{p}\right)-P_{y}\left(\lambda_{p}\right)\right] .
\end{aligned}
$$

Note that the coefficient in front of the power tuning term is $4 / 3$ vs the $1 / 3$ which occurs for the self-switching nonlinear rocking filter so that the switching power is reduced in this pump-signal geometry relative to that required for self-switching. ${ }^{7}$ Note also that the control beam powers $P_{x}$ and $P_{y}$ are independent of distance unless there is attenuation at the wavelength $\lambda_{p}$. It is the signal power which is interchanged between the $x$ and $y$ axes in the absence of the control beam. If the pump beam electric field polarization is incident at an angle $\phi$ to one of the fiber axes, the detuning $2 \delta$ at $\lambda_{s}=\lambda_{c}$ simplifies to $4 / 3 \gamma P_{p}[1,0,-1]$ for $\phi=\left[0^{\circ}, 45^{\circ}, 90^{\circ}\right]$. Therefore, maximum power-dependent detuning is expected for $\phi=0, \pi / 2$, and for $\phi=\pi / 4$ no effect on the signal is expected.

The equations describing the detailed evolution of the two polarization states for this case are an extension of those required for self-switching. ${ }^{7}$ They are

$$
-i \frac{d b_{x}}{d z}=\kappa b_{y}+\delta b_{x}, \quad-i \frac{d b_{y}}{d z}=\kappa b_{x}-\delta b_{y},
$$

where $\left|b_{x}(z)\right|^{2}$ and $\left|b_{y}(z)\right|^{2}$ are the powers of the $x$ - and $z$-polarized modes at position $z$. The coupling term $\kappa$ between the polarizations is $\pi / 2 L_{c}$ and $\delta$ is the detuning given by Eq. (4). Assuming that $b_{x}(0) \neq 0$ and $b_{y}(0)=0$, the solution for $z=L_{c}$ and $\lambda=\lambda_{c}$ is

$$
\frac{\left|b_{y}\left(L_{c}\right)\right|^{2}}{\left|b_{x}(0)\right|^{2}}=\left(\frac{\pi}{2}\right)^{2} \operatorname{sinc}^{2}\left\{\frac{\pi}{2}\left[1+\left(\frac{\delta}{\kappa}\right)^{2}\right]^{1 / 2}\right\}
$$

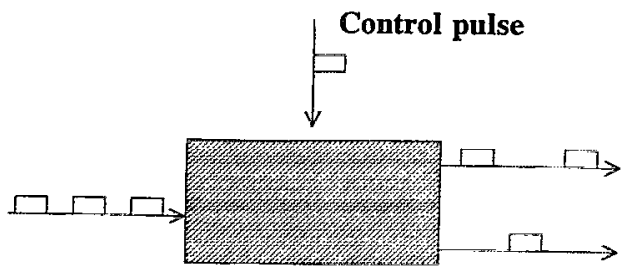

FIG. 1. Schematic geometry for demultiplexing a serial stream of signal pulses by a control (pump) pulse. 


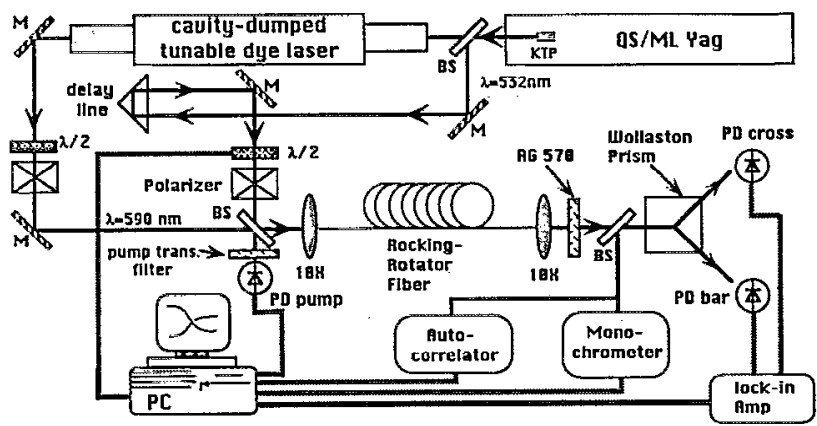

FIG. 2. The experimental apparatus used to demonstrate pump-signal demultiplexing.

where $\operatorname{sinc}(x)=\sin (x) / x$. The pump beam power, $P_{x}$ or $P_{y}$, required to completely inhibit the polarization rotation (which corresponds to $100 \%$ switching) is given by $3 \sqrt{ } 3 \kappa /$ $2 \gamma$, a reduction by a factor of 5.8 relative to the selfswitching case.

The experimental demonstration of pump-probe switching was carried out as follows. The rocking filter fiber was fabricated as discussed previously. ${ }^{6}$ Its center
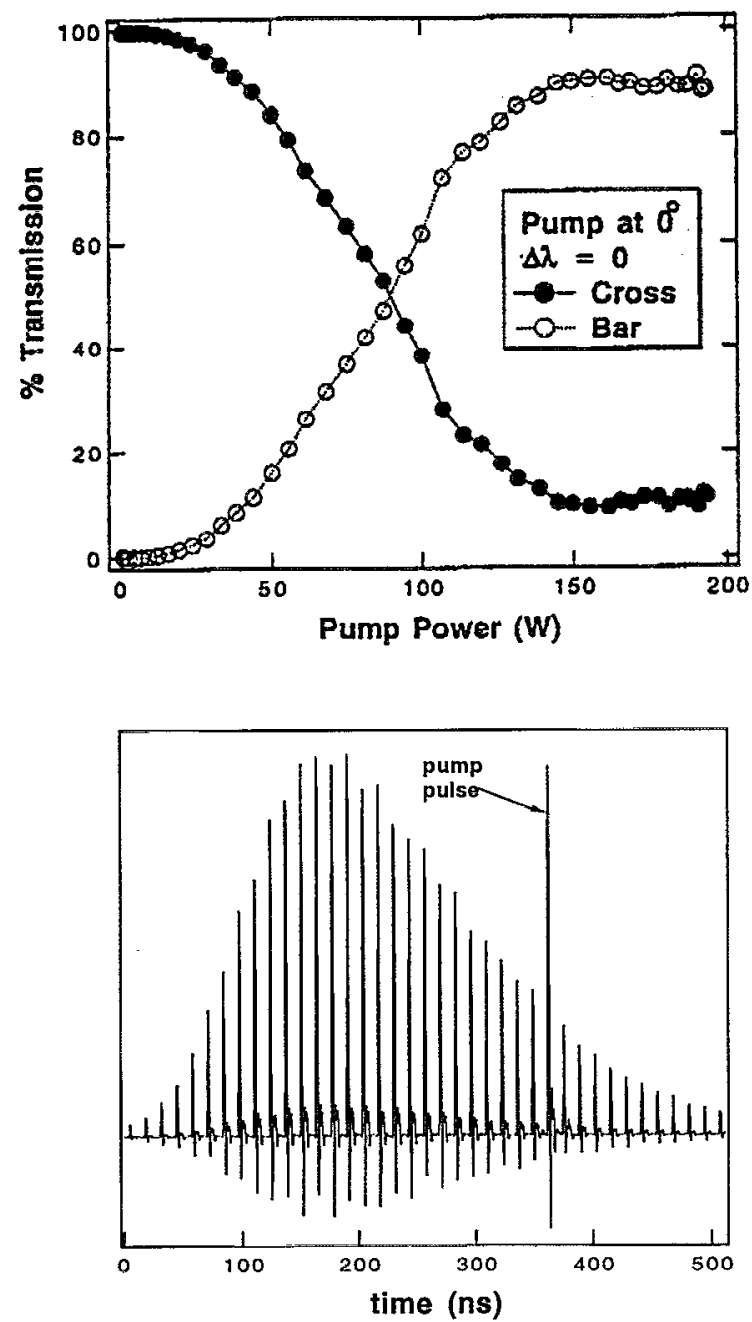

FIG. 3. The $Q$-switched envelope of mode-locked dye laser pulses, also showing the coincidence of the green pump pulse. wavelength was determined experimentally to be $590 \mathrm{~nm}$ with an effective length $L_{c}=127 \mathrm{~cm}$. The experimental apparatus is shown in Fig. 2. The experiments were implemented with 30 -ps signal pulses from a Coherent 740 dye laser of wavelength $590 \mathrm{~nm}$, pumped by a $Q$-switched, mode-locked Quantronix 416, doubled Nd:YAG laser. Shown in Fig. 3 is the signal $Q$-switched mode-locked pulse train and the pulse which is coincident with a single 80-ps green pulse from the doubled YAG used as the pump beam. The signal was input along one fiber polarization axis and the polarization direction of the pump pulses was varied from co-parallel to the signal polarization, to or-

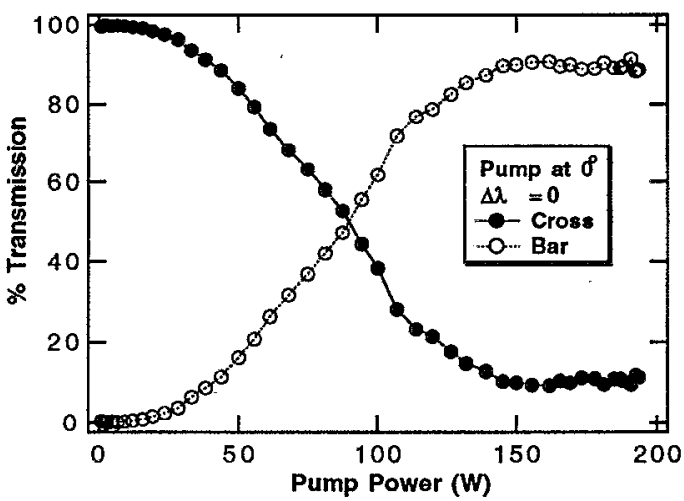

a)

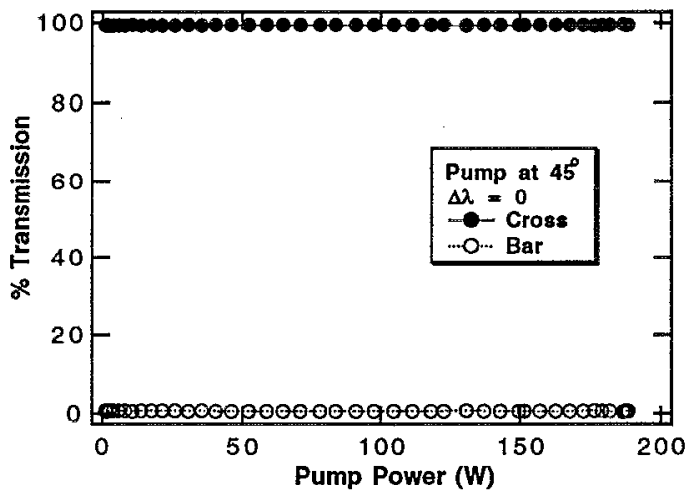

b)

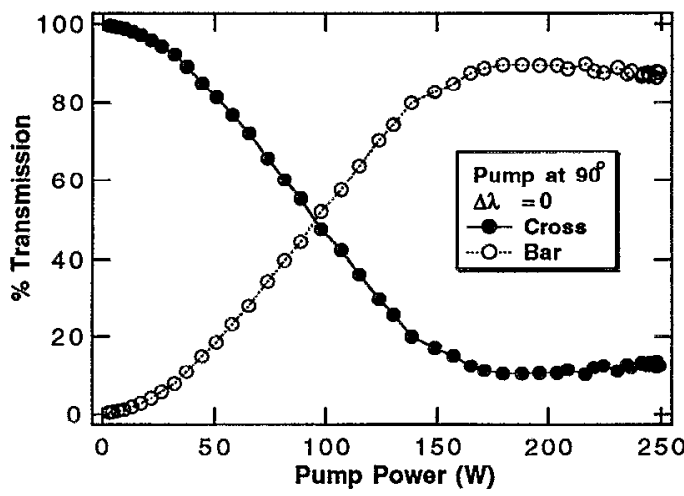

c)

FIG. 4. Pump induced switching of a signal beam as a function of peak pump-pulse power for different orientations of the pump beam polarization relative to the signal beam polarization. (a) Copolarized; (b) polarized at $45^{\circ} ;$ (c) orthogonally polarized. 
thogonal to it. After the fiber the pump signal was rejected by a wavelength-dependent beam splitter and the two orthogonally polarized signal outputs were detected on separate, slow photodiodes.

The fraction of the input energy of the switched-out ( $x$ polarized) signal pulse for three different polarizations of the pump beam relative to the signal beam are shown in Fig. 4. The fiber length was chosen to be $L_{c}$ to within a few percent so that in the absence of the pump, the signal power emerges totally from the cross channel (signal polarization rotated by $\pi / 2$ ). Maximum switching was obtained for the pump beam co-polarized with, and orthogonally polarized to the signal, as shown in Figs. 4(a) and $4(c)$. That is, with increasing pump power the output of the cross (bar) channel increased (decreased). For pump power polarized at $45^{\circ}$ to the fiber axes, no powerdependent switching was observed, all in excellent agreement with theory.

This approach to demultiplexing was found to be quite stable. The primary instability is the drift in the birefringence beat length with temperature which needs to be maintained to about $1^{\circ} \mathrm{C}$.

To obtain complete switching, the nonlinear wave vector detuning impressed onto the signal pulse by the pump pulse should ideally be a constant across the envelope of the signal pulse. This can be achieved by using a pump pulse temporarily much broader than the signal pulse and adjusting for peak pulse coincidence. Here, the ratio of the pump to signal pulse widths is $80 / 30$ which explains why the switching does not attain a full $100 \%$ but peaks instead at $90 \%$. However, this $90 \%$ is significantly better than the
$70 \%$ obtained previously for self-switching. ${ }^{8}$ Finally, the peak switching power was about $90 \mathrm{~W}$, greatly reduced from that observed previously for self-switching in this fiber. $^{8}$

Here, we have demonstrated the demultiplexing of a single pulse from a pulse train in the visible. Rocking filter fibers can also be fabricated with center wavelengths at 1300 and $1550 \mathrm{~nm}$, and $L_{c}=10-20 \mathrm{~m} .{ }^{9}$ An added advantage of using rotator fiber filters is their inherent stability to environmental fluctuations.

The CREOL part of the research was supported by AFOSR-90-086.

${ }^{1}$ M. Morioka, M. Saruwatari, and A. Takada, Electron. Lett. 23, 453 (1987); T. Morioka and M. Saruwatari, IEEE J. Selected Areas Commun. 6, 1186 (1988).

${ }^{2}$ K. J. Blow, N. J. Doran, B. K. Nayer, and B. P. Nelson, Opt. Lett. 15, 248 (1990).

${ }^{3}$ M. Jinno and T. Matsumoto, IEEE Photo. Technol. Lett. 2, 349 (1990); Electron. Lett. 27, 75 (1991).

${ }^{4}$ N. A. Whitaker, Jr., H. Avramopoulos, P. M. W. French, M. C. Gabriel, R. E. LaMarche, D. J. Di Giovanni, and H. M. Presby, Opt.Lett. 16, 1838 (1991).

${ }^{5}$ M. N. Islam, C. D. Poole, and J. P. Gordon, Opt. Lett. 14, 1011 (1989).

${ }^{6}$ R. H. Stolen, A. Ashkin, W. Pleibel, and J. M. Dziedzic, Opt. Lett. 9, 300 (1984).

${ }^{7}$ S. Trillo, S. Wabnitz, W. C. Banyai, N. Finlayson, C. T. Seaton, G. I. Stegeman, and R. H. Stolen, Appl. Phys. Lett. 53, 837 (1988); S. Trillo, S. Wabnitz, W. C. Banyai, N. Finlayson, C. T. Seaton, G. I. Stegeman, and R. H. Stolen, IEEE J. Quantum Electron. 25, 1042 (1989).

${ }^{8}$ C. G. Krautschik, G. I. Stegeman, and R. H. Stolen, Appl. Phys. Lett. 61, 1751 (1992).

${ }^{9}$ D. C. Johnson, F. Bilodeau, B. Malo, K. O. Hill, P. G. J. Wigley, and G. I. Stegeman, Opt. Lett. 17, 1635 (1992). 